\title{
BIBLIOGRAPHY
}

1. Gilbert Ames Bliss, A boundary value problem for a system of ordinary linear differential equations of the first order, Trans. Amer. Math. Soc. vol. 28 (1926) pp. 561584.

2. - Definitely self-adjoint boundary value problems, Trans. Amer. Math. Soc. vol. 44 (1938) pp. 413-428.

3. Augusto Bobonis, Differential systems with boundary conditions involving the characteristic parameter, Contributions to the calculus of variations, 1938-1941, pp. 103137.

4. William T. Reid, $A$ boundary value problem associated with the calculus of variations, Amer. J. Math. vol. 54 (1932) pp. 769-790.

5. - A system of ordinary linear differential equations with two-point boundary conditions, Trans. Amer. Math. Soc. vol. 44 (1938) pp. 508-521.

University of Puerto Rico

\section{ON THE SUMMATION OF MULTIPLE FOURIER SERIES. III ${ }^{1}$}

\section{K. CHANDRASEKHARAN}

Let $f(x)=f\left(x_{1}, \cdots, x_{k}\right)$ be a function of the Lebesgue class $L$, which is periodic in each of the $k$-variables, having the period $2 \pi$. Let

$$
\begin{aligned}
a_{\nu_{1}} \cdots \nu_{k} & =\frac{1}{(2 \pi)^{k}} \\
& \cdot \int_{-\pi}^{+\pi} \cdots \int_{-\pi}^{+\pi} f(x) \exp \left\{-i\left(\nu_{1} x_{1}+\cdots+\nu_{k} x_{k}\right)\right\} d x_{1} \cdots d x_{k},
\end{aligned}
$$

where $\left\{\nu_{k}\right\}$ are all integers. Then the series $\sum a_{\nu_{1}} \ldots \nu_{k} \exp i\left(\nu_{1} x_{1}+\cdots\right.$ $\left.+\nu_{k} x_{k}\right)$ is called the multiple Fourier series of the function $f(x)$, and we write

$$
f(x) \sim \sum a_{\nu_{1}} \ldots \nu_{k} \exp i\left(\nu_{1} x_{1}+\cdots+\nu_{k} x_{k}\right) .
$$

Let the numbers $\left(\nu_{1}{ }^{2}+\cdots+\nu_{k}^{2}\right)$, when arranged in increasing order of magnitude, be denoted by $\lambda_{0}<\lambda_{1}<\cdots<\lambda_{n}<\cdots$, and let

$$
C_{n}(x)=\sum a_{\nu_{1} \cdots \nu_{k}} \exp i\left(\nu_{1} x_{1}+\cdots+\nu_{k} x_{k}\right),
$$

where the sum is taken over all $\nu_{1}^{2}+\cdots+\nu_{k}{ }^{2}=\lambda_{n}$,

$$
\begin{aligned}
\phi(x, t) & =\sum C_{n}(x) \exp \left(-\lambda_{n} t\right), & \\
S_{R}(x) & =\sum_{\lambda_{n} \leqq R^{2}} C_{n}(x), & \lambda_{n} \leqq R^{2}<\lambda_{n+1} .
\end{aligned}
$$

Received by the editors December 7, 1945.

1 Papers I and II with the same title are to appear in Proc. London Math. Soc. 
Also, let $R_{k}\left(\lambda_{s}\right)$ and $r_{k}\left(\lambda_{s}\right)$ represent respectively the number of solutions of $\nu_{1}^{2}+\cdots+\nu_{k}^{2} \leqq \lambda_{8}$ and of $\nu_{1}^{2}+\cdots+\nu_{k}^{2}=\lambda_{8}$.

The object of this note is to study the convergence of multiple Fourier series, when summed up spherically by Bochner's method, that is, of the series $\sum C_{n}(x)$. We prove the following results.

THEOREM I. If

$$
\sum\left(\nu_{1}^{2}+\cdots+\nu_{k}^{2}\right)^{k / 2}\left|a_{\nu_{1}} \cdots \nu_{k}\right|^{2}<\infty,
$$

then the series $\sum_{n=0}^{\infty} C_{n}$ converges at every point of continuity of $f(x)$.

THEOREM II. If

$$
\sum\left(\nu_{1}^{2}+\cdots+\nu_{k}^{2}\right)^{k / 2+\bullet}\left|a_{\nu_{1}} \cdots \nu_{k}\right|^{2}<\infty, \quad \epsilon>0,
$$

then the series $\sum_{n=0}^{\infty} C_{n}$ converges absolutely.

The following result of Bochner ${ }^{2}$ is used in the proof of the above theorems.

LEMMA. At a point of continuity of $f(x), \phi(x, t)$ tends to a limit as $t$ tends to zero.

Proof of Theorem I. We shall first prove that

$$
\lim _{R \rightarrow \infty} S_{R}(x)=\lim _{t \rightarrow+0} \phi(x, t),
$$

whenever the limit on the right exists. Next, by the application of the above lemma, we deduce that at a point where $f(x)$ is continuous, $\sum C_{n}(x)$ is convergent.

Now

$$
\begin{aligned}
S_{R}(x)-\phi(x, t) & =\sum_{s=0}^{n} C_{s}\left[1-\exp \left(-\lambda_{s} t\right)\right]-\sum_{s=n+1}^{\infty} C_{s} \exp \left(-\lambda_{s} t\right) \\
& \equiv \sum J_{1}-J_{2},
\end{aligned}
$$

say. We have,

$$
\begin{aligned}
J_{1} & =\sum_{s=0}^{n} C_{s}\left[1-\exp \left(-\lambda_{s} t\right)\right] \\
& =\sum_{s=0}^{n}\left[1-\exp \left(-\lambda_{s} t\right)\right] \sum a_{\nu_{1}} \cdots \nu_{k} \exp \left[i\left(\nu_{1} x_{1}+\cdots+\nu_{k} x_{k}\right)\right] \\
& =\sum a_{\nu_{1}} \cdots \nu_{k} \exp i\left(\nu_{1} x_{1}+\cdots+\nu_{k} x_{k}\right)\left[1-\exp \left(-\nu_{1}^{2}-\cdots-\nu_{k}^{2}\right) t\right],
\end{aligned}
$$

2 S. Bochner, Summation of multiple Fourier series by spherical means, Trans. Amer. Math. Soc. vol. 40 (1936) pp. 175-207. 
where the third sum runs over $\lambda_{s}=\nu_{1}{ }^{2}+\cdots+\nu_{k}{ }^{2}$ and the last sum runs over $\nu_{1}^{2}+\cdots+\nu_{k}^{2} \leqq \lambda_{n}$, so that

$$
\begin{aligned}
\left|J_{1}\right| & \leqq \sum\left|a_{\nu_{1} \cdots \nu_{k}}\left\{1-\exp \left(-\nu_{1}^{2}-\cdots-\nu_{k}^{2}\right) t\right\}\right| \\
& \leqq\left[\sum\left(\nu_{1}^{2}+\cdots+\nu_{k}^{2}\right)^{k / 2}\left|a_{\nu_{1} \cdots \nu_{k}}\right|^{2}\right. \\
& \left.\times \sum\left\{1-\exp \left(-\nu_{1}^{2}-\cdots-\nu_{k}^{2}\right) t\right\}^{2}\left(\nu_{1}^{2}+\cdots+\nu_{k}^{2}\right)^{-k / 2}\right]^{1 / 2} \\
& \leqq O(1) \cdot t\left[\sum_{s=0}^{n} r_{k}\left(\lambda_{s}\right) \lambda_{s}^{2-k / 2}\right]^{1 / 2},
\end{aligned}
$$

where the first sum runs over $\nu_{1}^{2}+\cdots+\nu_{k}^{2} \leqq \lambda_{n}$.

Now,

$$
\begin{aligned}
\sum_{s=0}^{n} r_{k}\left(\lambda_{s}\right) \lambda_{s}^{2-k / 2} & =\sum_{s=0}^{n-1} R_{k}\left(\lambda_{s}\right)\left\{\lambda_{s}^{2-k / 2}-\lambda_{s+1}^{2-k / 2}\right\}+R_{k}\left(\lambda_{n}\right) \lambda_{n}^{2-k / 2} \\
& =O\left(\int_{0}^{\lambda_{n}} x d x\right)+O\left(\lambda_{n}^{2}\right) \\
& =O\left(\lambda_{n}^{2}\right)^{2}
\end{aligned}
$$

Hence, from (3), we obtain,

$$
\left|J_{1}\right|=O\left(\lambda_{n}\right)
$$

Again,

(6)

$$
\begin{aligned}
\left|J_{2}\right|= & \left|\sum_{s=n+1}^{\infty} C_{s} \exp \left(-\lambda_{s} t\right)\right| \\
\leqq & \lambda_{n}^{-k / 4} \sum_{s=n+1}^{\infty} \lambda_{s}^{k / 4}\left|C_{s} \exp \left(-\lambda_{s} t\right)\right| \\
\leqq & \quad \lambda_{n}^{-k / 4}\left[\sum\left(\nu_{1}^{2}+\cdots+\nu_{k}^{2}\right)^{k / 2}\left|a_{\nu_{1}} \cdots \nu_{k}\right|^{2}\right. \\
& \left.\quad \times \sum \exp \left\{-2\left(\nu_{1}^{2}+\cdots+\nu_{k}^{2}\right) t\right\}\right]^{1 / 2} \\
\leqq &
\end{aligned}
$$

(in the last two sums $\nu_{1}{ }^{2}+\cdots+\nu_{k}{ }^{2}$ runs from $\lambda_{n+1}$ to $\infty$ ), where

$$
\sum\left(\nu_{1}^{2}+\cdots+\nu_{k}^{2}\right)^{k / 2}\left|a_{v_{1}} \cdots \nu_{k}\right|^{2}=\epsilon_{n}
$$

$\left(\nu_{1}^{2}+\cdots+\nu_{k}^{2}\right.$ runs from $\lambda_{n+1}$ to $\left.\infty\right)$, and $\epsilon_{n} \rightarrow 0$ as $n \rightarrow \infty$, since $\sum e^{-2 \nu^{2} t}=O\left(t^{-1 / 2}\right)$ as $t \rightarrow 0$. Thus, we have, from (5) and (6), 


$$
S_{R}(x)-\phi(x, t)=O\left(t \lambda_{n}\right)+o\left[\epsilon_{n}^{1 / 2}\left(t \lambda_{n}\right)^{-k / 4}\right] .
$$

If $t$ is so chosen that $t \lambda_{n}=\delta_{n}=\epsilon_{n}{ }^{1 / k}$, then,

$$
S_{R}(x)-\phi(x, t)=O\left(\delta_{n}\right)+O\left(\epsilon_{n}^{1 / 2} \cdot-\delta_{n}^{k / 4}\right)=o(1), \quad \text { as } n \rightarrow \infty .
$$

Proof of Theorem II.

$$
\begin{aligned}
\sum\left|C_{s}(x)\right| \leqq & \sum\left|a_{\nu_{1}} \cdots \nu_{k}\right| \\
\leqq & \left\{\sum\left(\nu_{1}^{2}+\cdots+\nu_{k}^{2}\right)^{k / 2+\epsilon}\left|a_{\nu_{1} \cdots \nu_{k}}\right|^{2}\right\}^{1 / 2} \\
& \times\left\{\sum\left(\nu_{1}^{2}+\cdots+\nu_{k}^{2}\right)^{-k / 2-\epsilon}\right\}^{1 / 2} \\
= & O(1) \sum\left(r_{k}\left(\lambda_{s}\right) \lambda_{s}^{-k / 2-\epsilon}\right)^{1 / 2} \\
= & O\left(\left(\int^{\infty} R_{k}(x) x^{-k / 2-1-\epsilon} d x\right)^{1 / 2}\right) \\
= & O\left(\left(\int^{\infty} x^{-1-\epsilon} d x\right)^{1 / 2}\right)<\infty .
\end{aligned}
$$

On using Hölder's inequality instead of Schwarz's in (3) and (6), we can easily generalize Theorem I as follows:

If

$$
\sum\left(\nu_{1}^{2}+\cdots+\nu_{k}^{2}\right)^{k(p-1) / 2}\left|a_{\nu_{1}} \ldots \nu_{k}\right|^{p}<\infty,
$$

where $1<p \leqq 2$, then $\sum C_{n}$ converges at every point of continuity of $f(x)$.

MADRAS, INDIA 